\title{
The Association Between Oxidative Stress and Depressive Symptom Scores in Elderly Population: A Repeated Panel Study
}

\author{
Changwoo Han ${ }^{1}$, Youn-Hee Lim ${ }^{1,2}$, Yun-Chul Hong ${ }^{1,2,3}$ \\ ${ }^{1}$ Department of Preventive Medicine, Seoul National University College of Medicine, Seoul; ${ }^{2}$ Institute of Environmental Medicine, Seoul National \\ University Medical Research Center, Seoul; ${ }^{3}$ Environmental Health Center, Seoul National University College of Medicine, Seoul, Korea
}

Objectives: Previous epidemiological studies about oxidative stress and depression are limited by hospital-based case-control design, single-time measurements of oxidative stress biomarkers, and the small number of study participants. Therefore, in this study, we analyzed the association between biomarker of oxidative stress and depressive symptom scores using repeatedly measured panel data from a community-dwelling elderly population.

Methods: From 2008 to 2010, a total of 478 elderly participants residing in Seoul, Korea, were evaluated three times. Participants underwent the Korean version of the Short Form Generic Depression Scale (SGDS-K) test for screening depression, and urinary malondialdehyde (MDA) levels were measured as an oxidative stress biomarker. We used a generalized estimating equation with a compound symmetry covariance structure to estimate the effects of oxidative stress on depressive symptom scores.

Results: A two-fold increase in urinary MDA concentration was significantly associated with a 33.88\% (95\% confidence interval [Cl], $21.59 \%$ to $47.42 \%$ ) increase in total SGDS-K scores. In subgroup analyses by gender, a two-fold increase in urinary MDA concentration was significantly associated with increased SGDS-K scores in both men and women (men: $30.88 \%$; $95 \%$ Cl, $10.24 \%$ to $55.37 \%$; women: $34.77 \% ; 95 \% \mathrm{Cl}, 20.09 \%$ to $51.25 \%$ ). In bivariate analysis after an SGDS-K score $\geq 8$ was defined as depression, the third and the fourth urinary MDA quartiles showed a significantly increased odds ratio(OR) of depression compared to the lowest urinary MDA quartile (third quartile $\mathrm{OR}, 6.51 ; 95 \% \mathrm{Cl}, 1.77$ to 24.00 ; fourth quartile $\mathrm{OR}, 7.11 ; 95 \% \mathrm{Cl}, 1.99$ to 25.42 ).

Conclusions: Our study suggests a significant association between oxidative stress and depressive symptoms in the elderly population.

Key words: Depression, Depressive disorder, Oxidative stress, Malondialdehyde, Aged

Received: March 16, 2016 Accepted: August 5, 2016

Corresponding author: Yun-Chul Hong, MD, PhD

103 Daehak-ro, Jongno-gu, Seoul 03080, Korea

Tel: +82-2-740-8394, Fax: +82-2-747-4830

E-mail: ychong1@snu.ac.kr

This is an Open Access article distributed under the terms of the Creative Commons Attribution Non-Commercial License (http://creativecommons.org/licenses/by$\mathrm{nc} / 4.0 /$ ) which permits unrestricted non-commercial use, distribution, and reproduction in any medium, provided the original work is properly cited.

\section{INTRODUCTION}

Depression is a disease that poses a serious global public health burden. The World Health Organization has estimated that more than 350 million people suffer from depression worldwide, with nearly one million deaths due to depressionrelated suicide annually [1]. An enormous and increasing disease burden emphasizes the need to understand the etiologies and underlying pathogenic mechanisms of depression. 
Social, psychological, and biological risk factors for depression are believed to undergo a complex interaction in depression pathogenesis $[2,3]$. Interestingly, some of the risk factors for depression increase oxidative stress in human tissues [4,5], suggesting a common pathway between oxidative stress and depression.

Oxidative stress refers to an imbalanced state between free radical generation and antioxidant potential [6]. Oxidative phosphorylation from mitochondria produces not only adenosine triphosphate but also byproducts such as reactive oxygen species (ROS) and reactive nitrogen species. When the generation of free radicals exceeds the antioxidant capacity of the body, oxidative stress occurs and induces damage to all major groups of cellular macromolecules [7].

An association between oxidative stress and depression has been reported in various epidemiological, brain imaging, and molecular studies [4,8-11]. A recently published meta-analysis also showed a significant association between oxidative stress and depression [8]. In another systematic review, depression was significantly associated with an increase in the oxidative stress markers 8-hydroxydeoyguanosine and F2-isoprostanes [9]. Because oxidative stress is a major contributor to neuronal degeneration in the brain region, it is biologically plausible to explain a causal relationship between oxidative stress and depression [4].

However, many of the previous epidemiological studies between oxidative stress and depression have several limitations. First, most of the studies were performed without repeated measurements of oxidative stress biomarkers. A single-measurement protocol may limit the reliability of previous findings, since oxidative stress and depressive symptoms vary over time. Second, most of the previous studies have been hospitalbased case-control studies comparing the oxidative stress markers of patients with depression and those of healthy controls. Therefore, a dose-response relationship between oxidative stress and the severity of depressive symptoms in populations without clinically diagnosed depression could not be assessed [8]. Third, although the imbalance between oxygen radical generation and antioxidant potential increases with age, most of the studies are conducted in young adult populations with a small number of study participants [12].

Therefore, in this study, we analyzed the association between oxidative stress biomarker and depressive symptom scores from the panel data of community-dwelling elderly participants. Due to the changeable nature of oxidative stress and depressive symptoms, repeatedly measured data may reasonably capture the possible association. In addition, we evaluated the association in a population without clinically diagnosed depression. Finally, we focused on the elderly population because of their reduced capacity to respond to external stress and increased imbalance between radical generation and antioxidant potential compared to the younger population $[12,13]$. In other words, we analyzed the population that is most vulnerable to oxidative stress, making the observation of any association between oxidative stress and depressive symptoms more likely.

\section{METHODS}

\section{Study Population and Data Collection}

The Korean Elderly Environmental Panel (KEEP) study recruited 560 participants (aged $\geq 60$ years) to explore the relationship between environmental exposure and health outcomes in the elderly. A detailed description of the KEEP study design and methods is available elsewhere [14]. The study participants visited a community welfare center for medical examinations up to five times. During three examination cycles out of their total visits, urinary analysis and depression screening tests were performed at the same time, with an average 1-year follow-up interval. The first examination of urinary oxidative stress markers and depressive symptoms was conducted from September to December 2008. The second and third examinations were conducted from April to December 2009 and from March to August 2010, respectively (Supplemental Figure 1). We obtained detailed information about the participants using a structured questionnaire, including demographics, lifestyle habits, and medical history at the time of enrollment. The study protocol was approved by the institutional review board (IRB) at Seoul National University Hospital, Seoul, Korea (IRB no. H-0804-045-241).

Among the panel, we excluded those with missing urine samples or depression screening tests $(n=42)$. Because depression screening was assessed with a self-reported questionnaire, we excluded participants with low Mini Mental Status Examination scores because of concerns regarding the reliability of the depression screening tests $(n=13)$. In addition, to evaluate the association between oxidative stress markers and depression symptoms in a population without clinically diagnosed depression, we also excluded participants who were taking anti-depressant medications $(n=4)$. After further elimi- 


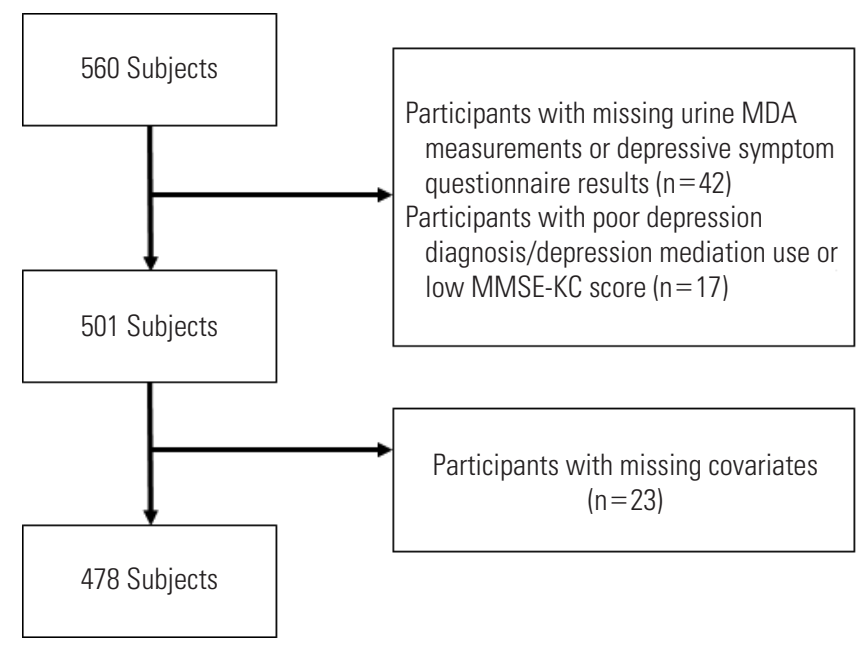

Figure 1. Flow diagram of study population. MDA, malondialdehyde; MMSE-KC, Mini-Mental Status Examination in the Korean version of the Consortium to Establish a Registry for Alzheimer's Disease Assessment Packet.

nating participants with missing covariates, a total of 917 observations from 478 participants were included in our analyses (Figure 1). Excluded participants were less likely to have a history of hypertension and consumed less alcohol. However, there were no statistically significant differences between the excluded and final study participants in other general characteristics (Supplemental Table 1). Out of our final study population, 127 participated in oxidative stress marker measurement and depressive symptom test three times, 185 participated two times, and 166 participated just one time.

\section{Urinary Malondialdehyde Measurement}

To assess oxidative stress in the central nervous system with neurons and high-lipid tissues, we used urinary malondialdehyde (MDA), which is a byproduct of polyunsaturated fatty acid peroxidation. Urinary MDA levels were determined by measuring thiobarbituric acid reactive substances. A sample of $50 \mu \mathrm{L}$ of urine was mixed with $300 \mu \mathrm{L}$ of $0.5 \mathrm{M}$ phosphoric acid solution and $150 \mu \mathrm{L}$ of $12 \mathrm{mM}$ TBA solution (T5500 SigmaAldrich, Sigma-Aldrich, Steinheim, Germany) and heated at $9^{\circ} \mathrm{C}$ for 1 hour. After cooling on ice, the mixture was vortexed with $500 \mu \mathrm{L}$ of methanol and centrifuged at $5000 \times \mathrm{g}$. Absorbance of the supernatant was measured at $532 \mathrm{~nm}$ using high-performance liquid chromatography and ultraviolet detection. The mobile phase was potassium phosphate $(0.05$ $\mathrm{mol} / \mathrm{L}, \mathrm{pH} 6.8)$ and methanol $(58: 42, \mathrm{v} / \mathrm{v})$. To minimize the influence of the urinary excretion rate, the urinary MDA concen- tration was adjusted by urinary creatinine ( $\mu \mathrm{g} / \mathrm{g}$ creatinine). Urinary creatinine levels were measured using a Hitachi 7600 instrument (Hitachi, Tokyo, Japan). Briefly, $10 \mathrm{~mL}$ of urine and $300 \mathrm{~mL}$ of picric acid solution (Wako, Osaka, Japan) were mixed at room temperature for three minutes, and absorbance of the solution was measured at $505 \mathrm{~nm}$. Following this, $75 \mathrm{~mL}$ of alkaline solution (Wako) were added for four minutes, and absorbance of the solution was measured at $570 \mathrm{~nm}$. The mean of the two values measured at 505 and $570 \mathrm{~nm}$ was used as the creatinine level.

\section{Measurement of Depressive Symptoms}

To evaluate the depressive symptoms during the previous week, we used the Korean version of the Short Form Generic Depression Scale (SGDS-K) questionnaire [15]. The Geriatric Depression Scale (GDS)-Short Form is a 15-item short version of the 30-item GDS [16]. Each of the 15 items on the SGDS-K is answered "yes" or "no," with the total score ranging from 0 to 15. The higher scores indicate more severe depressive symptoms. The SGDS-K is composed of three different components asking about emotional, somatic, and affective symptoms. Therefore, after conducting analysis on the total score, subgroup analyses for each component of the SGDS-K were also conducted. In addition, a prior validation study found that a cut-off score of eight or more achieved $85 \%$ sensitivity and $70 \%$ specificity for major depression [15]. Therefore, a participant who scored eight or more on the SGDS-K was defined as a depression case in bivariate analyses between urinary MDA quartile and risk of depression. To validate the reliability of the SGDS-K questionnaire response to some extent, we previously conducted test-retest comparisons among 30 randomly selected KEEP study participants [17]. By comparing two study visits with a 1-week interval, we found a high correlation of SGDS-K scores (Pearson $r=0.92$ ).

\section{Other Variables}

Information on age, gender, smoking status, alcohol consumption, education level, and history of hypertension and diabetes mellitus was collected with the structured questionnaire. Regular alcohol consumption was marked as "yes" for participants who had consumed alcohol at least once a month for 10 years or longer, and smoking status was classified according to never, former, and current smoker. Although primary and secondhand smoking is a major cause of oxidative stress, we had a limited number of smokers in the final study 
population. Therefore, we included creatinine-adjusted urinary cotinine levels in our analyses to adjust for the primary and secondhand smoke exposure of each participant. Urinary cotinine levels were analyzed with an enzyme-linked immunosorbent assay method [18]. Education level was classified as basic (less than primary education), medium (more than primary but less than secondary education), and high (secondary education or greater). Height and weight were measured at each time of visit and body mass index (BMI) was calculated as weight in kilograms divided by height in meters squared. Blood pressure was measured two times with an automatic sphygmomanometer (HEM-780, Omron, Kyoto, Japan) after 10 minutes of rest, with an interval of at least 10 minutes. We used the mean of both systolic blood pressure measurements in our analysis as covariates.

\section{Statistical Analysis}

Urinary MDA concentration was adjusted by urinary creatinine ( $\mu \mathrm{g} / \mathrm{g}$ creatinine) and log-transformed to approximate a normal distribution. Due to the panel design of the KEEP study, the number of study visits varied among the participants. If the number of study visits is not random, it may lead to a selection bias [19]. Therefore, we weighted subsequent visits from each participant using an inverse probability of attaining follow-up visits [20]. We used a $\chi^{2}$ test to compare the general characteristics between the final study population and excluded study participants. By using the initial visit data of final study participants, we calculated the arithmetic means of urinary MDA and their statistical values for each demographic characteristic using ANOVA.

Covariates that may have confounded the relationship between oxidative stress and depressive symptom scores were selected a priori and adjusted in the analyses. In model 1, participants' age, gender, history of diabetes mellitus, and education levels were adjusted. In model 2, we adjusted for model 1 variables plus history of hypertension, alcohol consumption, $\mathrm{BMI}$, fasting glucose, urinary cotinine, and systolic blood pressure. The initial covariates were selected based on an earlier literature review $[17,21,22]$. After applying bivariate analysis, only significant covariates ( $p$-value below 0.25 ) that predicted depressive symptoms at the first visit were selected for model 1 (Supplemental Table 2). In model 2, we adjusted for all the initial covariates selected for the analysis. Fasting glucose, BMI, urinary cotinine, and systolic blood pressure were included in the models as time-varying variables, while other covariates were included as time-independent.

The association between urinary MDA and depressive symptom scores was assessed with the following steps. First, to confirm a linear association between depressive symptom scores and urinary MDA concentration, non-parametric analysis using generalized additive mixed models (GAMM) was used. After assuming a linear association, a generalized estimating equation (GEE) was used to estimate the effects of oxidative stress on depressive symptom score. To account for the correlation within participants in repeated data analysis, we assumed a compound symmetry structure to give the same covariance regardless of the length of the time interval between the measurements. The Pearson correlation coefficient of two consecutive examinations within one participant was $0.37(p<0.01)$ for depressive symptom scores and $0.14(p<0.01)$ for urinary MDA concentration. We expressed the estimated effects of a twofold increase in urinary MDA levels as the percent change in depressive symptom scores using the following equation:

$$
\begin{aligned}
& \ln S G D S 1=\beta(\operatorname{InMDA}) \quad \operatorname{In} S G D S 2=\beta\left(\ln \left(M D A^{*} 2\right)\right) \\
& \ln S G D S 2 / S G D S 1=\beta(\operatorname{In} 2) \quad S G D S 2 / S G D S 1=2^{\beta} \\
& \text { Two-fold increase in urinary MDA levels causes } \\
& \left(2^{\beta}-1\right)^{*} 100 \% \text { changes in SGDS score }
\end{aligned}
$$

We also calculated the odds ratios (ORs) for risk of depression by urinary MDA quartiles using GEE analysis. The cut point for the quartile was $0.20,0.30$, and $0.42 \mu \mathrm{g} / \mathrm{g}$ creatinine for men and $0.25,0.35$, and $0.48 \mu \mathrm{g} / \mathrm{g}$ creatinine for women, respectively.

All analyses were performed using SAS version 9.4 (SAS Institute Inc., Cary, NC, USA) and R version 3.2.2 (The Comprehensive R Archive Network: http://cran.r-project.org). The statistical testing was conducted with a conventional two-tailed alpha level of 0.05 .

\section{RESULTS}

Study participants' characteristics at first visit and mean MDA levels are presented in Table 1. The mean age of the 478 study participants was 70.7 years and the mean BMI was 24.7 $\mathrm{kg} / \mathrm{m}^{2}$. Out of the participants, $74 \%$ were women and $87 \%$ were non-smokers. Women, non-drinkers, and thinner participants showed increased urinary MDA levels at first visit. In addition, the level of urinary MDA concentration was different according to educational level. 
Table 2 shows the average of SGDS-K scores and the subgroup scores for each component of the SGDS-K according to each examination cycle. The average SGDS-K score was 3.66 at the first examination period, 2.33 at the second, and 1.17 at the third examination period, showing a decreasing pattern. Similar patterns were observed in the emotional and affective components of the SGDS-K.

Figure 2 shows the non-parametric associations between urinary MDA concentration and SGDS-K score. The SGDS-K score increased with increasing concentrations of urinary MDA

Table 1. Demographic properties of the study population from the initial visit of participants $(n=478)$

\begin{tabular}{|c|c|c|c|}
\hline & n (\%) & $\begin{array}{c}\text { MDA levels } \\
\text { median /range } \\
\text { ( } \mu \mathrm{g} / \mathrm{g} \text { creatinine) }\end{array}$ & $p$-value ${ }^{1}$ \\
\hline \multicolumn{4}{|l|}{ Gender } \\
\hline Men & $124(25.94)$ & $0.34 / 0.03,1.92$ & $<0.01$ \\
\hline Women & $354(74.06)$ & $0.39 / 0.10,2.00$ & \\
\hline \multicolumn{4}{|l|}{ Age (y) } \\
\hline $60-69$ & $220(46.03)$ & $0.37 / 0.04,1.66$ & 0.58 \\
\hline $70-79$ & 237 (49.58) & $0.38 / 0.05,2.00$ & \\
\hline$\geq 80$ & $21(4.39)$ & $0.43 / 0.08,1.41$ & \\
\hline \multicolumn{4}{|c|}{ History of hypertension } \\
\hline No & $225(47.07)$ & $0.39 / 0.08,2.00$ & 0.19 \\
\hline Yes & $253(52.93)$ & $0.37 / 0.03,1.92$ & \\
\hline \multicolumn{4}{|c|}{ History of diabetes mellitus } \\
\hline No & 397 (83.05) & $0.38 / 0.03,1.92$ & 0.35 \\
\hline Yes & $81(16.95)$ & $0.38 / 0.05,2.00$ & \\
\hline \multicolumn{4}{|l|}{$\begin{array}{c}\text { Regular alcohol } \\
\text { consumption }\end{array}$} \\
\hline No & $364(76.15)$ & $0.39 / 0.04,2.00$ & $<0.01$ \\
\hline Yes & $114(23.85)$ & $0.36 / 0.03,1.16$ & \\
\hline \multicolumn{4}{|l|}{ Smoking status } \\
\hline Smoker & $28(5.86)$ & $0.35 / 0.10,0.75$ & 0.27 \\
\hline Ex-smoker & $33(6.90)$ & $0.36 / 0.04,0.80$ & \\
\hline Non-smoker & 417 (87.24) & $0.38 / 0.03,2.00$ & \\
\hline \multicolumn{4}{|l|}{ Education level } \\
\hline$<$ Primary & 138 (28.87) & $0.42 / 0.08,1.65$ & $<0.01$ \\
\hline Primary-Secondary & $213(44.56)$ & $0.39 / 0.04,2.00$ & \\
\hline$\geq$ Secondary & $127(26.57)$ & $0.33 / 0.03,1.66$ & \\
\hline \multicolumn{4}{|l|}{ BMI $\left(\mathrm{kg} / \mathrm{m}^{2}\right)$} \\
\hline$<25.0$ & $271(56.69)$ & $0.38 / 0.04,1.92$ & $<0.01$ \\
\hline $25.0-29.9$ & 188 (39.33) & $0.39 / 0.06,2.00$ & \\
\hline$\geq 30$ & 19 (3.97) & $0.32 / 0.03,0.69$ & \\
\hline
\end{tabular}

MDA, malondialdehyde; BMI, body mass index.

${ }^{1}$ Log-transformed arithmetic means of creatinine-adjusted urinary MDA and their significance for each demographic characteristic were assessed by ANOVA. and it showed an almost linear association. Therefore, after assuming linearity, we used a generalized estimating equation

Table 2. SGDS-K (depressive symptom) scores according to each examination cycle

\begin{tabular}{lcccc}
\hline & \multirow{2}{*}{$\begin{array}{c}\text { Total } \\
\text { score }\end{array}$} & \multicolumn{3}{c}{ Depressive symptom } \\
\cline { 3 - 5 } & & Emotional & Somatic & Affective \\
\hline 1st examination cycle & & & & \\
(Sep 2008-Dec 2008) & & & & \\
Total (n=277) & $3.66 \pm 3.33$ & $1.35 \pm 1.67$ & $0.69 \pm 0.80$ & $1.61 \pm 1.66$ \\
Men ( $n=60)$ & $3.33 \pm 3.15$ & $1.25 \pm 1.54$ & $0.53 \pm 0.77$ & $1.55 \pm 1.61$ \\
Women (n=217) & $3.75 \pm 3.38$ & $1.38 \pm 1.71$ & $0.73 \pm 0.81$ & $1.63 \pm 1.67$ \\
2nd examination cycle & & & & \\
(Apr 2009-Dec 2009) & & & & \\
Total (n=330) & $2.33 \pm 3.19$ & $0.80 \pm 1.43$ & $0.37 \pm 0.64$ & $1.16 \pm 1.61$ \\
Men (n=80) & $1.86 \pm 2.80$ & $0.64 \pm 1.21$ & $0.26 \pm 0.55$ & $0.96 \pm 1.37$ \\
Women (n=250) & $2.48 \pm 3.30$ & $0.86 \pm 1.49$ & $0.41 \pm 0.67$ & $1.22 \pm 1.67$ \\
3rd examination cycle & & & & \\
(Mar 2010-Aug 2010) & & & & \\
Total (n=310) & $1.17 \pm 2.40$ & $0.40 \pm 0.92$ & $0.38 \pm 0.87$ & $0.39 \pm 0.91$ \\
Men ( $n=84)$ & $0.57 \pm 1.40$ & $0.18 \pm 0.42$ & $0.18 \pm 0.62$ & $0.21 \pm 0.60$ \\
Women (n=226) & $1.39 \pm 2.64$ & $0.48 \pm 1.04$ & $0.46 \pm 0.93$ & $0.46 \pm 0.99$ \\
\hline
\end{tabular}

Values are presented as mean \pm standard deviation.

${ }^{1}$ Total Korean version of the Short Form Generic Depression Scale (SGDS-K) score was calculated by summing up each category of depressive symptom (emotional, somatic, and affective).

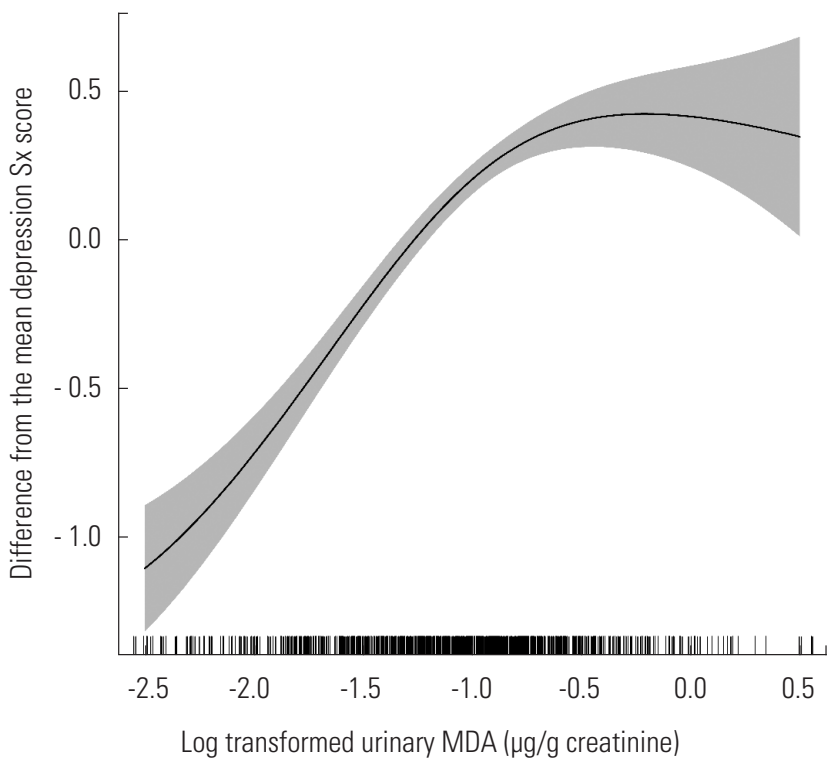

Figure 2. Non-parametric association between urinary malondialdehyde (MDA) concentration with depression symptom (Sx) score (results from generalized additive mixed models analysis). Data was adjusted for age, gender, history of hypertension and diabetes mellitus, alcohol consumption, educational level, body mass index, urinary cotinine, fasting glucose level, and systolic blood pressure. 
model in our analysis.

Figure 3 displays the estimated percent changes in SGDS-K score per two-fold increase in urinary MDA concentration. A two-fold increase in urinary MDA concentration was significantly associated with a $33.88 \%$ (95\% confidence interval [Cl], $21.59 \%$ to $47.42 \%$ ) increase in the total SGDS-K score (Supple-

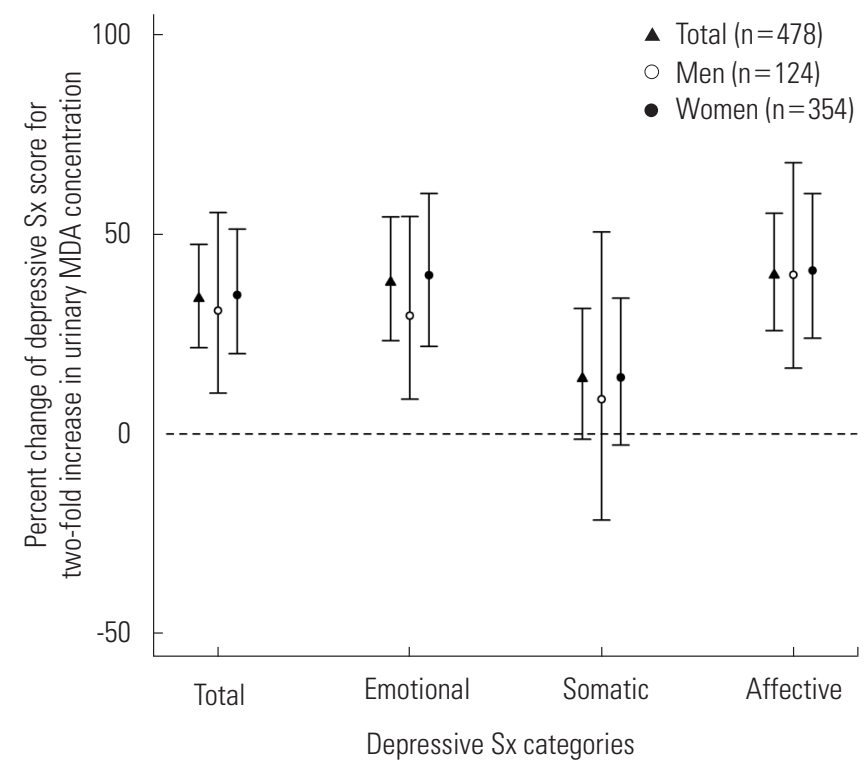

Figure 3. Percent change in Korean version of the Short Form Generic Depression Scale scores per two-fold increase in urinary malondialdehyde (MDA) concentration (results from generalized estimating equation analysis). Data was adjusted for age, gender, history of hypertension and diabetes mellitus, alcohol consumption, educational level, body mass index, urinary cotinine, fasting glucose level, and systolic blood pressure. Sx, symptom. mental Table 3). In subgroup analyses, a two-fold increase in urinary MDA concentration was significantly associated with increased SGDS-K score in both men and women (men: $30.88 \%$; $95 \% \mathrm{Cl}, 10.24 \%$ to $55.37 \%$; women: $34.77 \%$, $95 \% \mathrm{Cl}$, $20.09 \%$ to $51.25 \%)$. In analysis by each component of the SGDS-K score, we found a significant association between urinary MDA concentration with emotional and affective symptom scores, but not with somatic symptom scores. A two-fold increase in urinary MDA concentration was associated with a $37.96 \%(95 \% \mathrm{Cl}, 23.34 \%$ to $54.30 \%), 13.89 \%$ ( $95 \% \mathrm{Cl},-1.31 \%$ to $31.42 \%)$, and $39.76 \%(95 \% \mathrm{Cl}, 25.85 \%$ to $55.22 \%)$ increase in emotional, somatic, and affective symptom scores, respectively. Similar patterns were observed in subgroup analyses by gender. In men, a two-fold increase in urinary MDA concentration was associated with a $29.59 \%(95 \% \mathrm{Cl}, 8.74 \%$ to $54.43 \%)$, $8.69 \%(95 \% \mathrm{Cl},-21.53 \%$ to $50.55 \%)$, and $39.81 \%(95 \% \mathrm{Cl}$, $16.48 \%$ to $67.82 \%$ ) increase in emotional, somatic, and affective symptom scores, respectively. In women, a two-fold increase in urinary MDA concentration was associated with a $39.73 \%$ ( $95 \% \mathrm{Cl}, 21.91 \%$ to $60.15 \%), 14.15 \%$ ( $95 \% \mathrm{Cl},-2.76 \%$ to $34.00 \%)$, and $40.88 \%(95 \% \mathrm{Cl}, 23.96 \%$ to $60.12 \%)$ increase in emotional, somatic, and affective symptom scores, respectively.

Table 3 shows the ORs and 95\% Cls for depression according to the quartile of the urinary MDA levels. Compared to the lowest urinary MDA quartile, we found a statistically significant increase in depression risk in the third and the fourth quartiles (third quartile: $\mathrm{OR}, 6.51 ; 95 \% \mathrm{Cl}, 1.77$ to 24.00 ; fourth quartile: $\mathrm{OR}, 7.11 ; 95 \% \mathrm{Cl}, 1.99$ to 25.42$)$. We also found a significant OR when we restricted the analyses to women. Although we could not find a significant association in analysis

Table 3. Adjusted odds ratio for depression according to urinary malondialdehyde levels

\begin{tabular}{|c|c|c|c|c|c|}
\hline & No. of events & 10 & 20 & 30 & 40 \\
\hline \multicolumn{6}{|l|}{ Total } \\
\hline No. of observations & 917 & 229 & 245 & 221 & 222 \\
\hline Depression $^{2}(+) /(-)$ & $81 / 836$ & 1.00 (reference) & $3.78(1.01,14.03)$ & $6.51(1.77,24.00)$ & $7.11(1.99,25.42)$ \\
\hline \multicolumn{6}{|l|}{ Men } \\
\hline No. of observations & 224 & 54 & 56 & 58 & 56 \\
\hline Depression (+)/(-) & $14 / 210$ & 1.00 (reference) & $2.76(0.33,23.41)$ & $2.87(0.34,24.54)$ & $3.06(0.51,18.42)$ \\
\hline \multicolumn{6}{|l|}{ Women } \\
\hline No. of observations & 693 & 177 & 164 & 180 & 172 \\
\hline Depression (+)/(-) & $67 / 626$ & 1.00 (reference) & $5.26(1.23,22.42)$ & $7.49(1.70,33.11)$ & $8.36(1.92,36.63)$ \\
\hline
\end{tabular}

Values are presented as odds ratio (95\% confidence interval).

0 , quartile; GEE, generalized estimating equation.

${ }^{1}$ Adjusted for age, gender, history of hypertension and diabetes mellitus, alcohol consumption, educational level, body mass index, urinary cotinine, fasting glucose level, and systolic blood pressure (results from GEE analysis).

${ }^{2} \mathrm{~A}$ participant who scored eight or more on the Korean version of the Short Form Generic Depression Scale test was defined as a depression case. 
with men, the associations were still positive and increased in a dose-dependent fashion.

\section{DISCUSSION}

We observed a significant association between oxidative stress marker and depressive symptom scores in our analysis with the elderly panel. A two-fold increase in urinary MDA concentration was associated with an approximately $30 \%$ increase in depressive symptom scores and similar findings were noted in several subgroup analyses. In the bivariate analysis, after SGDS-K score $\geq 8$ was defined as depression, we found a statistically significant increase of depression risk in the third and the fourth MDA quartiles compared to the lowest MDA quartile.

Epidemiological, brain imaging, postmortem, and molecular studies suggest a possible association between oxidative stress and depression. Most of the case-control studies have found increased oxidative stress markers in depression patients compared to healthy controls. In a recent meta-analysis and systematic review of previous studies, a significant association between oxidative stress and depression was suggested $[8,9]$. Brain imaging studies and postmortem studies showed reduced volume of the prefrontal cortex, hippocampus, and a reduced number of glial cells in depression patients, which supports the notion that oxidative stress-related apoptosis is involved in depression pathogenesis [4]. By comparing ROS detoxification enzyme levels in recurrent depression patients and healthy controls, increased superoxide dismutase was noticed in depression patients' frontal brain tissue [10]. Reduced gene expression of metallothionein $1 \mathrm{M}$ protein, which regulates oxidative stress, has also been noted in the prefrontal cortex of major depression patients [11].

However, only a few studies have evaluated oxidative stress and the severity of depressive symptom in participants without clinically diagnosed depression [23,24]. In addition, as far as we know, only one study has evaluated the association between oxidative stress and depression among older persons aged over 60 [25]. In our study, we found a significant association between oxidative stress and depressive symptom scores among older individuals without clinically diagnosed depression. Moreover, the degree of the depressive symptom score was linearly associated with MDA levels in women, thus showing a dose-response relationship (Supplemental Figure 2). A non-linear association in the results of GAMM analysis in men seems to have originated from the relatively small number of observations compared to women (number of observation used in men: 224; women: 693). However, in additional analysis assuming a non-linear association, we found results similar to those in our main analysis (results not shown).

Although many studies indicate an intimate association between oxidative stress and depression, the direction of the association is still unclear. In other words, oxidative stress may work as a key pathogenic mediator of depression, but increased oxidative stress could also be an outcome of depression. For example, major depression is characterized by an elevation in immune activation, which leads to excessive production of ROS [26,27]. Increased catecholamine metabolism in depression patients also induces lipid peroxidation and ROS production $[28,29]$. Decreased oxidative stress parameters after the introduction of depression medication could be another reason to regard oxidative stress as an outcome of depression [30,31].

However, oxidative stress has received attention as a possible pathogenic mediator in the development of various diseases [32]. In the same fashion, many researchers have suggested oxidative stress as one of the possible pathogenic mediators for depression for the following biological reasons. First, many of the risk factors for depression have been shown to cause changes in oxidative stress markers. For example, psychosocial stress, substance abuse, aging, and environmental factors such as air pollution and various chemicals that have been found to be associated with depression may induce oxidative stress in various human tissues [5,33-35]. Second, the brain is regarded as one of the tissues most vulnerable to oxidative stress. Due to the high oxygen state and modest antioxidant defense mechanisms, oxidative stress could be intensified in the brain lesion [5]. In addition, there are abundant lipid substrates such as polyunsaturated fatty acid (PUFA) for oxidation and various ion chemicals of the brain may work as catalase for free radical reaction [36]. Therefore, oxidative stress may easily impact neuronal cell functions of the brain.

Third, increased oxidative stress could show several characteristic features that underlie the pathogenesis of clinical depression. ROS induces oxidation of PUFA and reduced PUFA levels are believed to contribute to depression pathophysiology [37]. After exposure to ROS, protein undergoes deterioration, which may lead to changes in tertiary structure or enzymatic activity [38]. For instance, the number of glycoprotein neural cell adhesion molecules (NCAM) decreases at the sur- 
face of neuronal and glial cells after oxidative stress exposure [39]. In line with this study, NCAM-knockout mice show decreased hippocampal neurogenesis and depression-like symptoms [40].

Therefore, an association between oxidative stress and depression is apparent despite the undetermined exact causal direction. Oxidative stress and depression may be involved in a complex vicious cycle, in which each one reinforces the other. Thus, by avoiding an imbalance between free radical generation and antioxidant potential, we may reduce the occurrence or exacerbation of depression.

To our knowledge, this is the first panel study to report an association between oxidative stress levels and depressive symptoms in the elderly population. By analyzing repeatedly measured data from older persons without clinical depression, our results may support the findings from previous studies. Regarding the constantly changing properties of oxidative stress and depressive symptoms, panel data analysis may provide higher levels of evidence in the association between oxidative stress and depression. Furthermore, the self-reported depression questionnaire asked about depressive symptoms within the past week. Because MDA is short-term marker of oxidative stress, we may reasonably speculate about an association between short-term oxidative stress exposure and depressive symptoms.

However, there are several limitations of this study that must be noted. First, the use of MDA as the only oxidative stress biomarker may limit our findings. MDA is a short-term oxidative stress marker that is susceptible to confounders such as dietary antioxidants. However, because the central nervous system is composed of neurons and high lipid tissues, MDA, the byproduct of polyunsaturated fatty acid peroxidation, may reasonably represent oxidative stress of the brain. Second, although we adjusted for various time-dependent and independent factors in the analytical models, other potential confounders may have been overlooked. Third, the excluded study participants had different demographic characteristics (less history of hypertension and consumption of alcohol) compared to final study participants. Therefore, a potential selection bias may have occurred in our study. In addition, KEEP study participants were elderly people residing in Seoul, Korea. Therefore, our findings may not be generalizable to the elderly population in other cities or countries.

In conclusion, our study suggests a positive association between oxidative stress and depressive symptoms in an elderly population without clinically diagnosed depression.

\section{ACKNOWLEDGEMENTS}

We thank all the study participants, research workers, and technicians who contributed to this study. This study was supported by the Women Scientist Research Program through the National Research Foundation of Korea (NRF) funded by the Ministry of Science, ICT, and Future Planning (\#2015R1A1A3A 04001325).

\section{CONFLICT OF INTEREST}

The authors have no conflicts of interest associated with the material presented in this paper.

\section{ORCID}

Changwoo Han http://orcid.org/0000-0002-5127-4680

Youn-Hee Lim http://orcid.org/0000-0002-1290-5814

Yun-Chul Hong http://orcid.org/0000-0001-9010-7271

\section{REFERENCES}

1. Marcus M, Yasamy MT, van Ommeren M, Chisholm D, Saxena S; WHO Department of Mental Health and Substance Abuse. Depression: a global public health concern; 2012 [cited 2016 Sep 5]. Available from: http://www.who.int/mental_health/ management/depression/who_paper_depression_wfmh_ 2012.pdf?ua $=1$.

2. Fiske A, Wetherell JL, Gatz M. Depression in older adults. Annu Rev Clin Psychol 2009;5:363-389.

3. Reeve J, Lloyd-Williams M, Dowrick C. Revisiting depression in palliative care settings: the need to focus on clinical utility over validity. Palliat Med 2008;22(4):383-391.

4. Michel TM, Pülschen D, Thome J. The role of oxidative stress in depressive disorders. Curr Pharm Des 2012;18(36):5890-5899.

5. Hovatta I, Juhila J, Donner J. Oxidative stress in anxiety and comorbid disorders. Neurosci Res 2010;68(4):261-275.

6. Halliwell B, Gutteridge JM. Free radicals in biology and medicine. Oxford: Oxford University Press; 2015, p. 199-200.

7. Fridovich I. Biological effects of the superoxide radical. Arch Biochem Biophys 1986;247(1):1-11.

8. Palta P, Samuel LJ, Miller ER 3rd, Szanton SL. Depression and oxidative stress: results from a meta-analysis of observational 
studies. Psychosom Med 2014;76(1):12-19.

9. Black CN, Bot M, Scheffer PG, Cuijpers P, Penninx BW. Is depression associated with increased oxidative stress? A systematic review and meta-analysis. Psychoneuroendocrinology 2015;51:164-175.

10. Michel TM, Frangou S, Thiemeyer D, Camara S, Jecel J, Nara K, et al. Evidence for oxidative stress in the frontal cortex in patients with recurrent depressive disorder--a postmortem study. Psychiatry Res 2007;151(1-2):145-150.

11. Shelton RC, Claiborne J, Sidoryk-Wegrzynowicz M, Reddy R, Aschner M, Lewis DA, et al. Altered expression of genes involved in inflammation and apoptosis in frontal cortex in major depression. Mol Psychiatry 2011;16(7):751-762.

12. Sohal RS, Weindruch R. Oxidative stress, caloric restriction, and aging. Science 1996;273(5271):59-63.

13. Risher JF, Todd GD, Meyer D, Zunker CL. The elderly as a sensitive population in environmental exposures: making the case. Rev Environ Contam Toxicol 2010;207:95-157.

14. Han C, Lim YH, Hong YC. Does cadmium exposure contribute to depressive symptoms in the elderly population? Occup Environ Med 2016;73(4):269-274.

15. Bae JN, Cho MJ. Development of the Korean version of the Geriatric Depression Scale and its short form among elderly psychiatric patients. J Psychosom Res 2004;57(3):297-305.

16. Yesavage JA, Sheikh J. 9/Geriatric Depression Scale (GDS) recent evidence and development of a shorter violence. Clin Gerontol 1986;5(1-2):165-173.

17. Lim YH, Kim H, Kim JH, Bae S, Park HY, Hong YC. Air pollution and symptoms of depression in elderly adults. Environ Health Perspect 2012;120(7):1023-1028.

18. Kim JH, Choi YH, Bae S, Park HY, Hong YC. eNOS gene polymorphisms modify the association of $\mathrm{PM}(10)$ with oxidative stress. Toxicol Lett 2012;214(3):263-267.

19. Rubin DB. Inference and missing data. Biometrika 1976;63(3): 581-592.

20. Robins JM, Rotnitzky A, Zhao LP. Analysis of semiparametric regression models for repeated outcomes in the presence of missing data. J Am Stat Assoc 1995;90(429):106-121.

21. Kim BS, Bae JN, Cho MJ. Depressive symptoms in elderly adults with hypotension: different associations with positive and negative affect. J Affect Disord 2010;127(1-3):359-364.

22. Golden SH, Lazo M, Carnethon M, Bertoni AG, Schreiner PJ, Diez Roux AV, et al. Examining a bidirectional association between depressive symptoms and diabetes. JAMA 2008;299 (23):2751-2759.
23. Kobrosly R, van Wijngaarden E. Associations between immunologic, inflammatory, and oxidative stress markers with severity of depressive symptoms: an analysis of the 2005-2006 National Health and Nutrition Examination Survey. Neurotoxicology 2010;31(1):126-133.

24. Zhou F, Zhang W, Wei Y, Zhou D, Su Z, Meng X, et al. The changes of oxidative stress and human 8-hydroxyguanine glycosylase1 gene expression in depressive patients with acute leukemia. Leuk Res 2007;31(3):387-393.

25. Dimopoulos N, Piperi C, Psarra V, Lea RW, Kalofoutis A. Increased plasma levels of 8-iso-PGF2alpha and IL-6 in an elderly population with depression. Psychiatry Res 2008;161(1):5966.

26. Kook Al, Mizruchin A, Odnopozov N, Gershon H, Segev Y. Depression and immunity: the biochemical interrelationship between the central nervous system and the immune system. Biol Psychiatry 1995;37(11):817-819.

27. Raison CL, Capuron L, Miller AH. Cytokines sing the blues: inflammation and the pathogenesis of depression. Trends Immunol 2006;27(1):24-31.

28. Hibbeln JR, Salem N Jr. Dietary polyunsaturated fatty acids and depression: when cholesterol does not satisfy. Am J Clin Nutr 1995;62(1):1-9.

29. Maher P, Davis JB. The role of monoamine metabolism in oxidative glutamate toxicity. J Neurosci 1996;16(20):6394-6401.

30. Cumurcu BE, Ozyurt H, Etikan I, Demir S, Karlidag R. Total antioxidant capacity and total oxidant status in patients with major depression: impact of antidepressant treatment. Psychiatry Clin Neurosci 2009;63(5):639-645.

31. Kotan VO, Sarandol E, Kirhan E, Ozkaya G, Kirli S. Effects of long-term antidepressant treatment on oxidative status in major depressive disorder: a 24-week follow-up study. Prog Neuropsychopharmacol Biol Psychiatry 2011;35(5):12841290.

32. Valko M, Leibfritz D, Moncol J, Cronin MT, Mazur M, Telser J. Free radicals and antioxidants in normal physiological functions and human disease. Int J Biochem Cell Biol 2007;39(1): 44-84.

33. Gidron Y, Ronson A. Psychosocial factors, biological mediators, and cancer prognosis: a new look at an old story. Curr Opin Oncol 2008;20(4):386-392.

34. Chuang KJ, Chan CC, Su TC, Lee CT, Tang CS. The effect of urban air pollution on inflammation, oxidative stress, coagulation, and autonomic dysfunction in young adults. Am J Respir Crit Care Med 2007;176(4):370-376. 
35. Hong YC, Park EY, Park MS, Ko JA, Oh SY, Kim H, et al. Community level exposure to chemicals and oxidative stress in adult population. Toxicol Lett 2009;184(2):139-144.

36. Evans $\mathrm{PH}$. Free radicals in brain metabolism and pathology. $\mathrm{Br}$ Med Bull 1993;49(3):577-587.

37. Bakunina N, Pariante CM, Zunszain PA. Immune mechanisms linked to depression via oxidative stress and neuroprogression. Immunology 2015;144(3):365-373.

38. Kohen R, Nyska A. Oxidation of biological systems: oxidative stress phenomena, antioxidants, redox reactions, and meth- ods for their quantification. Toxicol Pathol 2002;30(6):620-650.

39. Fujita-Hamabe W, Tokuyama S. The involvement of cleavage of neural cell adhesion molecule in neuronal death under oxidative stress conditions in cultured cortical neurons. Biol Pharm Bull 2012;35(4):624-628.

40. Aonurm-Helm A, Berezin V, Bock E, Zharkovsky A. NCAM-mimetic, FGL peptide, restores disrupted fibroblast growth factor receptor (FGFR) phosphorylation and FGFR mediated signaling in neural cell adhesion molecule (NCAM)-deficient mice. Brain Res 2010;1309:1-8. 
Supplemental Table 1. General characteristics of study participants across final study population and excluded participants

\begin{tabular}{|c|c|c|c|}
\hline & $\begin{array}{c}\text { Final study } \\
\text { population } \\
(n=478)\end{array}$ & $\begin{array}{c}\text { Excluded } \\
\text { study partici- } \\
\text { pants }(n=82)\end{array}$ & $p$-value \\
\hline \multicolumn{4}{|l|}{ Gender } \\
\hline Men & $124(25.94)$ & $22(26.83)$ & 0.87 \\
\hline Women & $354(74.06)$ & $60(73.17)$ & \\
\hline \multicolumn{4}{|l|}{ Age (y) } \\
\hline $60-69$ & $220(46.03)$ & $27(36.59)$ & 0.15 \\
\hline $70-79$ & 237 (49.58) & $44(60.98)$ & \\
\hline$\geq 80$ & $21(4.39)$ & $2(2.44)$ & \\
\hline \multicolumn{4}{|c|}{ History of hypertension } \\
\hline No & $225(47.07)$ & $52(63.41)$ & $<0.01$ \\
\hline Yes & $253(52.93)$ & 30 (36.59) & \\
\hline \multicolumn{4}{|c|}{ History of diabetes mellitus } \\
\hline No & $397(83.05)$ & $72(87.80)$ & 0.28 \\
\hline Yes & $81(16.95)$ & $10(12.20)$ & \\
\hline \multicolumn{4}{|c|}{ Regular alcohol consumption } \\
\hline No & $364(76.15)$ & 58 (89.23) & 0.02 \\
\hline Yes & $114(23.85)$ & $7(10.77)$ & \\
\hline Did not answer & & $\mathrm{n}=17$ & \\
\hline \multicolumn{4}{|l|}{ Smoking status } \\
\hline Smoker & $28(5.86)$ & $3(4.41)$ & 0.79 \\
\hline Ex-smoker & $33(6.90)$ & $3(4.41)$ & \\
\hline Non-smoker & $417(87.24)$ & 62 (91.18) & \\
\hline Did not answer & & $n=14$ & \\
\hline \multicolumn{4}{|l|}{ Education level } \\
\hline$<$ Primary & 138 (28.87) & $17(25.76)$ & 0.78 \\
\hline Primary-Secondary & $213(44.56)$ & $29(43.94)$ & \\
\hline$\geq$ Seondary & $127(26.57)$ & $20(30.30)$ & \\
\hline Did not answer & & $n=16$ & \\
\hline \multicolumn{4}{|c|}{ Body mass index $\left(\mathrm{kg} / \mathrm{m}^{2}\right)$} \\
\hline$<25.0$ & $271(56.69)$ & $45(54.88)$ & 0.19 \\
\hline $25.0-29.9$ & 188 (39.33) & $30(36.59)$ & \\
\hline$\geq 30$ & $19(3.97)$ & $7(8.54)$ & \\
\hline
\end{tabular}

Values are presented as number (\%). 
Journal of

Preventive Medicine \& Public Health

Supplemental Table 2. Demographic properties of the study population from the first visit of participants $(n=478)$

\begin{tabular}{|c|c|c|c|}
\hline & $\begin{array}{c}\text { Depression (-) } \\
\quad(n=421)\end{array}$ & $\begin{array}{l}\text { Depression (+) } \\
\quad(n=57)\end{array}$ & $p$-value ${ }^{1}$ \\
\hline \multicolumn{4}{|l|}{ Gender } \\
\hline Men & $113(26.84)$ & $11(19.30)$ & 0.22 \\
\hline Women & $308(73.16)$ & $46(80.70)$ & \\
\hline \multicolumn{4}{|l|}{ Age (y) } \\
\hline $60-69$ & $199(47.27)$ & $21(36.84)$ & 0.03 \\
\hline $70-79$ & $207(49.17)$ & $30(52.63)$ & \\
\hline$\geq 80$ & $15(3.56)$ & $6(10.53)$ & \\
\hline \multicolumn{4}{|l|}{ History of hypertension } \\
\hline No & $198(47.03)$ & 27 (47.37) & 0.96 \\
\hline Yes & $223(52.97)$ & $30(52.63)$ & \\
\hline \multicolumn{4}{|l|}{ History of diabetes } \\
\hline No & $353(83.85)$ & $44(77.19)$ & 0.21 \\
\hline Yes & $68(16.15)$ & $13(22.81)$ & \\
\hline \multicolumn{4}{|c|}{ Regular alcohol consumption } \\
\hline No & $318(75.53)$ & $46(80.70)$ & 0.39 \\
\hline Yes & $103(24.47)$ & $11(19.30)$ & \\
\hline \multicolumn{4}{|l|}{ Smoking status } \\
\hline Smoker & $24(5.70)$ & $4(7.02)$ & 0.86 \\
\hline Ex-smoker & $30(7.13)$ & $3(5.26)$ & \\
\hline Non-smoker & $367(87.17)$ & $50(87.72)$ & \\
\hline \multicolumn{4}{|l|}{ Education level } \\
\hline$<$ Primary & $114(27.08)$ & $24(42.11)$ & 0.02 \\
\hline Primary-Secondary & $188(44.66)$ & $25(43.86)$ & \\
\hline$\geq$ Secondary & $119(28.27)$ & $8(14.04)$ & \\
\hline \multicolumn{4}{|c|}{ Body mass index $\left(\mathrm{kg} / \mathrm{m}^{2}\right)$} \\
\hline$<25.0$ & $235(55.82)$ & $36(63.16)$ & 0.42 \\
\hline $25.0-29.9$ & 170 (40.38) & $18(31.85)$ & \\
\hline$>30$ & $16(3.80)$ & $3(5.26)$ & \\
\hline
\end{tabular}

${ }^{1} p$-values are calculated by $\chi^{2}$ test. 
Supplemental Table 3. Associations of the log-transformed urinary concentration of MDA with depressive Sx scores (results from GEE analysis)

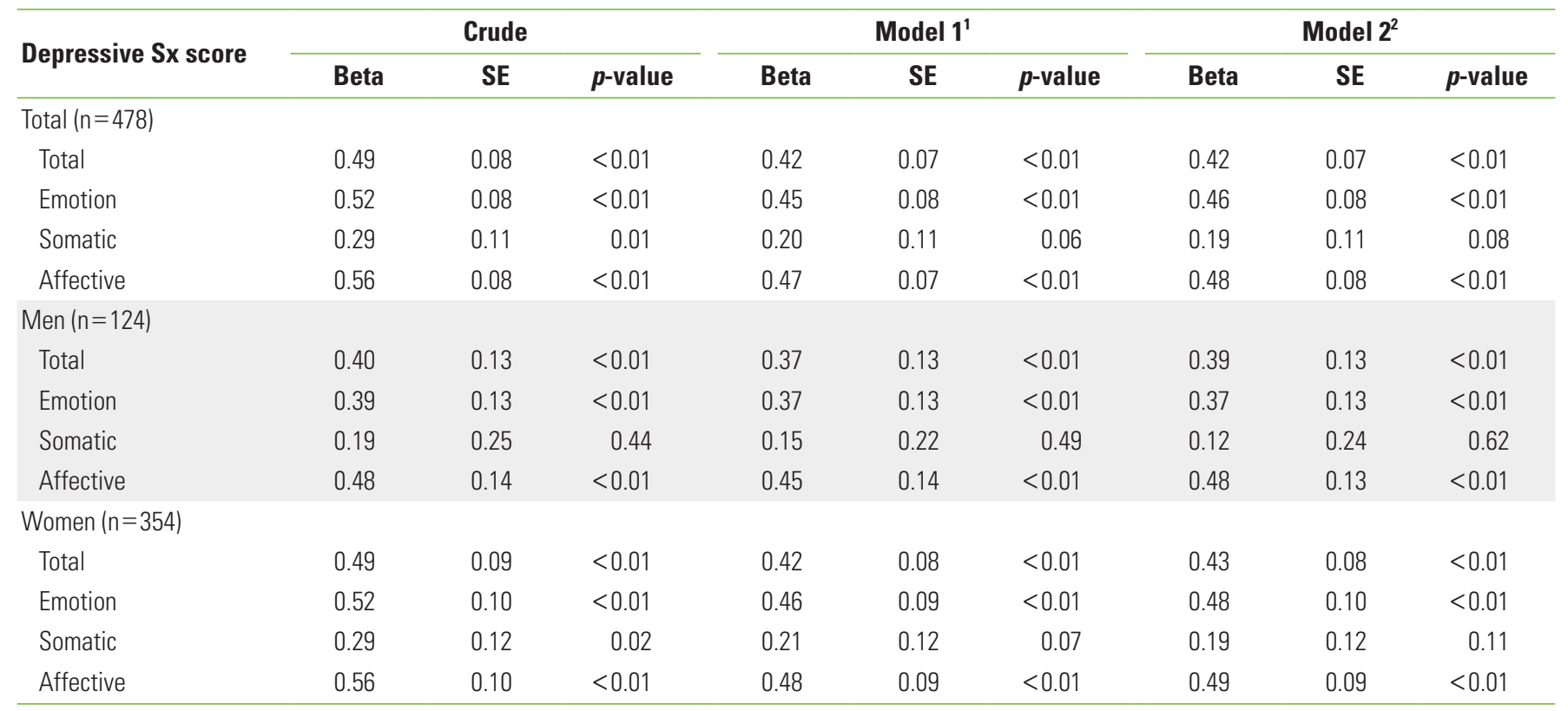

MDA, malondialdehyde; GEE, generalized estimating equation; Sx, symptom; SE, standard error.

${ }^{1}$ Model 1 was adjusted for age, gender, history of diabetes mellitus, and educational level.

${ }^{2}$ Model 2 was adjusted for model 1 puls history of hypertension, alcohol consumption, body mass index, fasting glucose, urinary cotinine, and systolic blood pressure. 


\section{Journal of}

Preventive Medicine \& Public Health

\begin{tabular}{|c|c|c|c|}
\hline No. & $\begin{array}{l}\text { 1st examination cycle } \\
\text { (Sep 2008-Dec 2008) }\end{array}$ & $\begin{array}{l}\text { 2nd examination cycle } \\
\text { (Apr 2009-Dec 2009) }\end{array}$ & $\begin{array}{l}\text { 3rd examination cycle } \\
\text { (Mar 2010-Aug 2010) }\end{array}$ \\
\hline \multirow{2}{*}{408} & & & Maximum 3 visits \\
\hline & Study enrollment & & \\
\hline \multicolumn{4}{|r|}{ Maximum 2 visits } \\
\hline 109 & & Study enrollment & \\
\hline \multicolumn{4}{|r|}{ Maximum 1 visit } \\
\hline 43 & & & Study enrollment \\
\hline
\end{tabular}

Supplemental Figure 1. Detailed structure and depression/urinary malondialdehyde examination cycle of Korean Elderly Environmental Panel study $(n=560)$. 

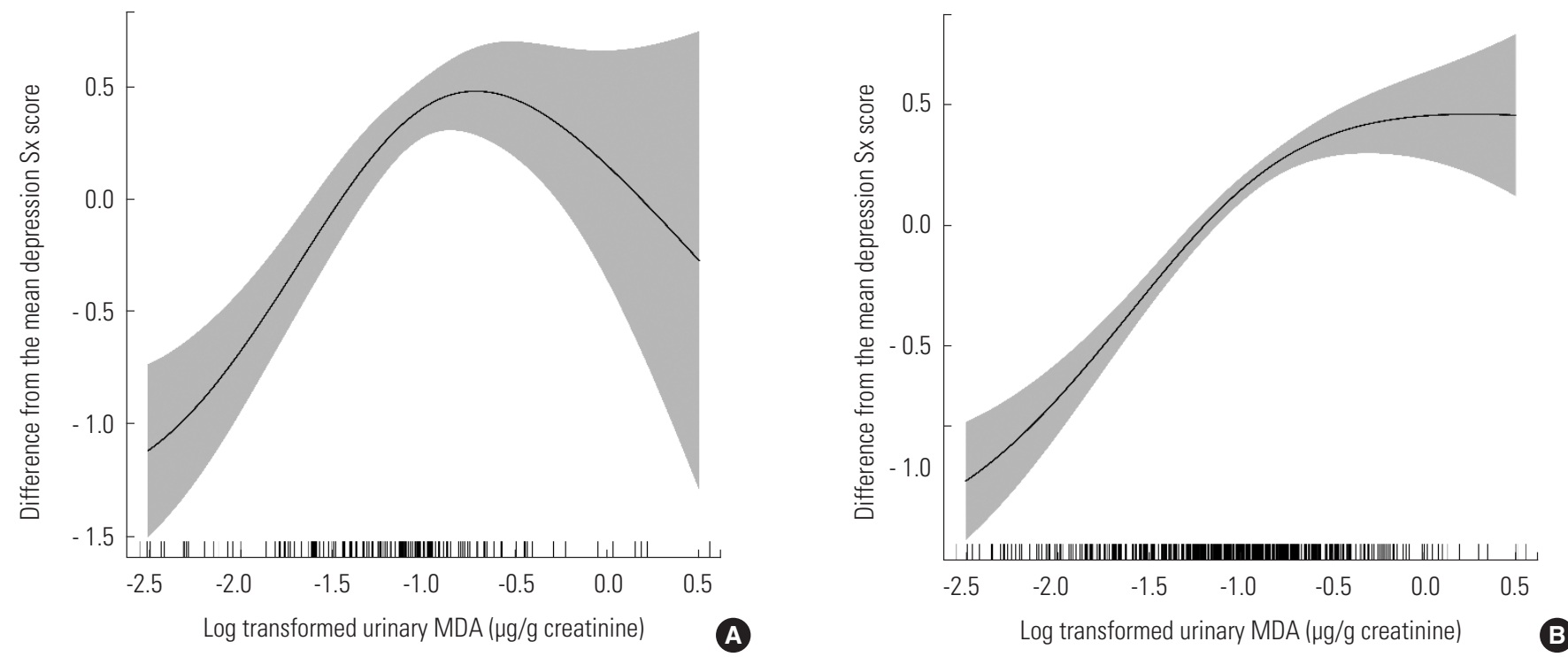

Supplemental Figure 2. Non-parametric association between urinary malondialdehyde (MDA) concentration with depression symptom (Sx) score (results of generalized additive mixed models analysis). (A) Men, (B) women. Data was adjusted for age, history of hypertension and diabetes mellitus, alcohol consumption, educational level, body mass index, urinary cotinine, fasting glucose level, and systolic blood pressure. 